\title{
DERIVED INVARIANCE OF SUPPORT VARIETIES
}

\author{
JULIAN KÜLSHAMMER, CHRYSOSTOMOS PSAROUDAKIS, \\ AND ØYSTEIN SKARTSÆTERHAGEN
}

\begin{abstract}
The (Fg) condition on Hochschild cohomology as well as the support variety theory are shown to be invariant under derived equivalence.
\end{abstract}

\section{INTRODUCTION}

In 1983, Carlson Car83 introduced the concept of the support variety of a module for group algebras using ordinary cohomology. This concept and its generalisations to other classes of algebras led to several interesting applications including for example a criterion for the representation type of an algebra [Far07], and connections to vector bundles [FP11]. In 2004, Snashall and Solberg [SS04] extended the theory to arbitrary finite dimensional algebras using Hochschild cohomology, and together with Erdmann, Holloway and Taillefer $\left[\mathrm{EHS}^{+}\right.$04] they introduced certain finiteness assumptions in order to obtain a theory of support varieties with good properties. These finiteness assumptions are called the (Fg) condition. The motivation for this article is the following question.

Question. Which types of equivalences between two algebras preserve the (Fg) condition, and more generally support varieties?

One answer to this question was given in [Lin11, Theorem 4.1]. Therein, Linckelmann has shown that the $(\mathrm{Fg})$ condition is stable under separable equivalence provided both algebras are symmetric. In particular this includes the cases of Morita equivalences, derived equivalences, and stable equivalences of Morita type (in the case of symmetric algebras). It seems to be well-known to the experts that the (Fg) condition is stable under Morita equivalence but the authors weren't able to find a reference for this fact (which will also follow from our main theorem).

Since the support variety of any bounded complex of projectives vanishes, a natural guess would also be that an equivalence of the singularity categories $\mathcal{D}^{b}(A) / \mathcal{K}^{b}(\operatorname{proj} A)$ yields invariance of support varieties. However, in joint work of the two last named authors with Øyvind Solberg [PSS14, Example 5.5], a counterexample is provided. The last named author of this article has shown that a singular equivalence of Morita type (with level)

Date: October 13, 2018.

2010 Mathematics Subject Classification. Primary 16E40, 16E65, 18E30; Secondary 16G10 .

Key words and phrases. Finite generation condition, Hochschild cohomology, Support varieties, Derived equivalences.

The authors want to thank Hongxing Chen, Steffen Koenig and Øyvind Solberg for useful discussions and valuable comments. These thanks are extended to Wei Hu for pointing out Remark 3.6. 
preserves (Fg) provided both algebras are known to be Gorenstein [Ska16]. This also includes the case of stable equivalence of Morita type for self-injective algebras. Here we show the following:

Theorem. Let $A$ and $B$ be derived equivalent $k$-algebras. Then the following hold.

(a) The $(\mathrm{Fg})$ condition holds for $A$ if and only if it holds for $B$.

(b) If $F: \mathcal{D}^{b}(A) \rightarrow \mathcal{D}^{b}(B)$ is a derived equivalence, then the support varieties of $M^{*}$ and $F\left(M^{*}\right)$ are isomorphic for every bounded complex of A-modules $M^{*}$.

Using the result in Ska16] part (a) can also be obtained as follows: The first step is to show that if $A$ satisfies $(\mathrm{Fg})$, then $A$ and $B$ are both Gorenstein. The algebra $A$ is Gorenstein by $\left[\mathrm{EHS}^{+} 04\right.$, Proposition 1.2]. The property of algebras being Gorenstein is preserved under derived equivalence by combining results of Happel [Hap91, Lemma 1.5] and Rickard [Ric89, Theorem 6.4, Proposition 9.1]. Hence, $B$ is also Gorenstein. Part (a) of the Theorem now follows by using that any derived equivalence induces a singular equivalence of Morita type with level [Wan14, Theorem 2.3] and that a singular equivalence of Morita type with level preserves (Fg) provided both algebras are Gorenstein [Ska16.

The structure of the article is as follows. Section 2 recalls the relevant parts of the theory of derived equivalences. Section 3 recalls the definition of a support variety for complexes and proves the Theorem. In the last section we give an example illustrating that our result can be applied to show ( Fg) for an algebra where previous criteria did not work.

Conventions and Notation: Throughout the article let $k$ be a field. We assume all $k$-algebras to be finite dimensional except for the subalgebras of the Hochschild cohomology ring. For a $k$-algebra $A$ we write $\bmod A$ for the category of finitely generated left $A$-modules, and $\operatorname{proj} A$ for the subcategory consisting of projective modules. We write $\mathcal{D}^{b}(A)$ for the bounded derived category of $\bmod A$ and $\mathcal{D}^{-}(A)$ for the derived category of bounded above complexes. We denote the homotopy category of bounded above complexes of projectives by $\mathcal{K}^{-}(\operatorname{proj} A)$ and its subcategory of complexes with bounded homology by $\mathcal{K}^{-, b}(\operatorname{proj} A)$. There is a triangle equivalence $N_{A}: \mathcal{K}^{-}(\operatorname{proj} A) \rightarrow \mathcal{D}^{-}(A)$ which restricts to an equivalence $N_{A}: \mathcal{K}^{-, b}(\operatorname{proj} A) \rightarrow \mathcal{D}^{b}(A)$. For complexes $X^{*}$ and $Y^{*}$ of $A$ modules we define $\operatorname{Hom}_{\mathcal{D}^{b}(A)}^{*}\left(X^{*}, Y^{*}\right):=\bigoplus_{n \in \mathbb{Z}} \operatorname{Hom}_{\mathcal{D}^{b}(A)}\left(X^{*}, Y^{*}[n]\right)$ and $\operatorname{End}_{\mathcal{D}^{b}(A)}^{*}\left(X^{*}\right):=$ $\operatorname{Hom}_{\mathcal{D}^{b}(A)}^{*}\left(X^{*}, X^{*}\right)$. Furthermore we denote by $A^{e}:=A \otimes_{k} A^{\text {op }}$ the enveloping algebra of $A$. The Hochschild cohomology ring is defined as $\operatorname{HH}^{*}(A):=\operatorname{End}_{\mathcal{D}^{b}\left(A^{e}\right)}^{*}(A)$. If char $k \neq 2$, let $\mathrm{HH}^{\mathrm{ev}}(A):=\mathrm{HH}^{2 *}(A)$, if char $k=2$, let $\operatorname{HH}^{\mathrm{ev}}(A):=\mathrm{HH}^{*}(A)$.

\section{Derived EQUivalences}

In this section, we state the definitions and results we need regarding derived categories and derived equivalences. We recall Rickard's derived analogue of Morita's theorem and as an application the invariance of Hochschild cohomology under derived equivalences. These results were first proved in [Ric89] and Ric91]. For an overview of Morita theory for derived categories, see the books [Zim14 and [KZ98]. Furthermore, we show that in our setting, the derived tensor product is associative. This is a fact that should be well-known, but for which we could not find a precise reference in the literature. 
Definition 2.1. Two $k$-algebras $A$ and $B$ are derived equivalent if there exists a triangle equivalence $F: \mathcal{D}^{b}(A) \rightarrow \mathcal{D}^{b}(B)$. The functor $F$ is then a derived equivalence between $A$ and $B$, and it is of standard type if $F \cong X^{*} \otimes_{A}^{\mathbb{L}}-$ for a complex $X^{*}$ of $A$-B-bimodules.

The following is part of Rickard's celebrated version of Morita's theorem:

Theorem 2.2. Let $A$ and $B$ be k-algebras. Let $F: \mathcal{D}^{b}(A) \rightarrow \mathcal{D}^{b}(B)$ be an equivalence. Then there is an equivalence of standard type $X^{*} \otimes_{A}^{\mathbb{L}}-: \mathcal{D}^{b}(A) \rightarrow \mathcal{D}^{b}(B)$ such that $F\left(M^{*}\right) \cong X^{*} \otimes_{A}^{\mathbb{L}} M^{*}$ for every $M^{*} \in \mathcal{D}^{b}(A)$. Its quasi-inverse is given by the equivalence of standard type $Y^{*} \otimes_{B}^{\mathbb{L}}-$, where $Y^{*}=\mathbb{R} \operatorname{Hom}_{A}\left(X^{*}, A\right)$.

This theorem allows us to always work with equivalences of standard type instead of general derived equivalences. Rickard also proved that such an equivalence gives a standard equivalence of the corresponding enveloping algebras, and hence an isomorphism of Hochschild cohomology rings:

Theorem 2.3. Let $A$ and $B$ be $k$-algebras. Suppose there is a derived equivalence of standard type $X^{*} \otimes_{A}^{\mathbb{L}}-: \mathcal{D}^{b}(A) \rightarrow \mathcal{D}^{b}(B)$, and let $Y^{*} \otimes_{B}^{\mathbb{L}}-$ be an inverse equivalence. Then we have the following.

(1) The functor $Y^{*} \otimes_{B}^{\mathbb{L}}\left(-\otimes_{B}^{\mathbb{L}} X^{*}\right): \mathcal{D}^{b}\left(B^{e}\right) \rightarrow \mathcal{D}^{b}\left(A^{e}\right)$ is a derived equivalence of standard type, and there is an isomorphism $\psi: Y^{*} \otimes_{B}^{\mathbb{L}} X^{*} \rightarrow A$ in $\mathcal{D}^{b}\left(A^{e}\right)$.

(2) Let $\psi_{*}: \operatorname{End}_{\mathcal{D}^{b}\left(A^{e}\right)}^{*}\left(Y^{*} \otimes_{B}^{\mathbb{L}} X^{*}\right) \rightarrow \mathrm{HH}^{*}(A)$, given by $\eta \mapsto \psi \eta \psi^{-1}$, be the isomorphism of endomorphism rings induced by $\psi$. Then the map

$$
\psi_{*} \circ\left(Y^{*} \otimes_{B}^{\mathbb{L}}\left(-\otimes_{B}^{\mathbb{L}} X^{*}\right)\right): \operatorname{HH}^{*}(B) \rightarrow \operatorname{HH}^{*}(A)
$$

is an isomorphism of graded $k$-algebras.

The following lemma states that when we are working with finite-dimensional $k$-algebras, the derived tensor product $\otimes^{\mathbb{L}}$ is associative (up to natural isomorphism).

Lemma 2.4. Let $A, B, C$ and $D$ be k-algebras, and let ${ }_{A} L_{B}^{*},{ }_{B} M_{C}^{*}$ and ${ }_{C} N_{D}^{*}$ be bounded complexes of bimodules. Then there is an isomorphism $\left(L^{*} \otimes_{B}^{\mathbb{L}} M^{*}\right) \otimes_{C}^{\mathbb{L}} N^{*} \cong L^{*} \otimes_{B}^{\mathbb{L}}\left(M^{*} \otimes_{C}^{\mathbb{L}}\right.$ $\left.N^{*}\right)$ which is natural in all three factors. More precisely, the following diagram of functors commutes up to natural isomorphism:

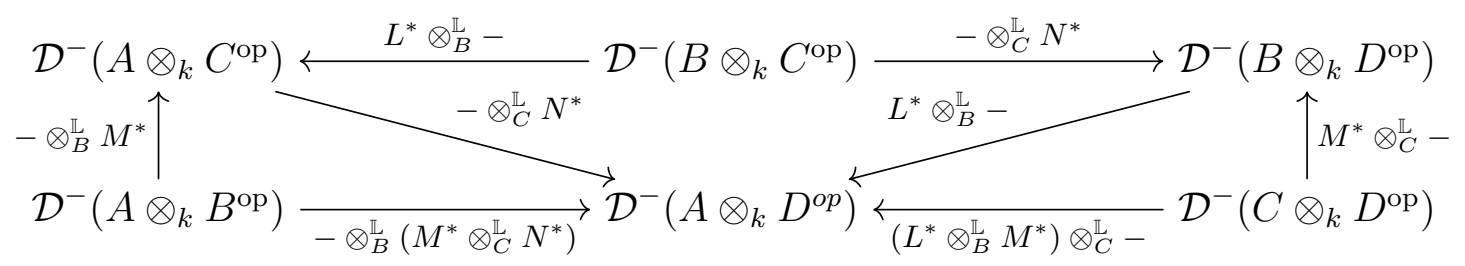

Proof. First recall that $-\otimes_{B}^{\mathbb{L}}-: \mathcal{D}^{-}\left(A \otimes_{k} B^{\text {op }}\right) \times \mathcal{D}^{-}\left(B \otimes_{k} C^{\text {op }}\right) \rightarrow \mathcal{D}^{-}\left(A \otimes_{k} C^{\text {op }}\right)$ is a bifunctor. This is noted as Exercise 10.6.2 in [Wei94]. For a sketch of proof in a slightly different situation we refer the reader to [HTT08, Appendix B]. Using this fact we can replace every module by its projective bimodule resolution: 
Consider the derived tensor functor $L^{*} \otimes_{B}^{\mathbb{L}}-$. Let $P_{L^{*}}$ be a projective bimodule resolution of $L^{*}$. Since there is a quasi-isomorphism $P_{L^{*}} \rightarrow L^{*}$ and $-\otimes_{B}^{\mathbb{L}}-$ is a bifunctor, we have a natural isomorphism $L^{*} \otimes_{B}^{\mathbb{L}}-\cong P_{L^{*}} \otimes_{B}^{\mathbb{L}}-$.

Next we claim that if $P^{*}$ is a bounded above complex of projective $A$ - $B$-bimodules, then $P^{*} \otimes_{B}^{\mathbb{L}}-=P^{*} \otimes_{B}-: \mathcal{D}^{-}\left(B \otimes_{k} C^{\text {op }}\right) \rightarrow \mathcal{D}^{-}\left(A \otimes_{k} C^{\text {op }}\right)$. It suffices to show that the functor $P^{*} \otimes_{B}-$ preserves acyclic complexes. Let $Q^{*}$ be an acyclic complex of $B$ - $C$-bimodules. Using the duality $\operatorname{Hom}_{k}(-, k)$ and the Hom-tensor adjunction we obtain an isomorphism

$$
\mathcal{H} \operatorname{om}_{k}\left(P^{*} \otimes_{B} Q^{*}, k\right) \cong \mathcal{H} \operatorname{om}_{B_{\text {op }}}\left(P^{*}, \operatorname{Hom}_{k}\left(Q^{*}, k\right)\right),
$$

where $\mathcal{H}$ om denotes the Hom complex: Recall that for two complexes $\left(M^{*}, d_{M}\right),\left(N^{*}, d_{N}\right)$ of $A$-modules, $\mathcal{H} \operatorname{om}\left(M^{*}, N^{*}\right)$ is the complex with $n$-th component $\prod_{i \in \mathbb{Z}} \operatorname{Hom}\left(M^{i}, N^{i+n}\right)$ and differential given by $\partial f=d_{N} \circ f-(-1)^{n} f \circ d_{M}$ for a homogeneous $f$ of degree $n$. Taking homology it follows that

$$
\begin{aligned}
H_{n}\left(\mathcal{H o m}_{k}^{*}\left(P^{*} \otimes_{B} Q^{*}, k\right)\right) & =H_{n}\left(\mathcal{H o m}_{B^{\mathrm{op}}}\left(P^{*}, \operatorname{Hom}_{k}\left(Q^{*}, k\right)\right)\right) \\
& \cong \operatorname{Hom}_{\mathcal{K}^{-}\left(B^{\mathrm{op}}\right)}\left(P^{*}, \operatorname{Hom}_{k}\left(Q^{*}, k\right)[n]\right)=0
\end{aligned}
$$

for any $n$. Note that the last Hom space is zero because $P^{*}$ is a complex of projectives and $\operatorname{Hom}_{k}\left(Q^{*}, k\right)[n]$ is an acyclic complex. This implies that the complex $P^{*} \otimes_{B} Q^{*}$ is acyclic. This finishes the proof of the claim. By combining the two steps, we get a natural isomorphism $L^{*} \otimes_{B}^{\mathbb{L}}-\cong P_{L^{*}} \otimes_{B}-$.

We can describe the other functors similarly. For the functor $\left(L^{*} \otimes_{B}^{\mathbb{L}} M^{*}\right) \otimes_{C}^{\mathbb{L}}-$, we need a projective bimodule resolution of $L^{*} \otimes_{B}^{\mathbb{L}} M^{*}$. We claim that $P_{L^{*}} \otimes_{B} P_{M^{*}}$ is such a resolution, where $P_{L^{*}}$ and $P_{M^{*}}$ are projective bimodule resolutions of $L^{*}$ and $M^{*}$, respectively. Using the above description of the functor $L^{*} \otimes_{B}^{\mathbb{L}}-$, we have $L^{*} \otimes_{B}^{\mathbb{L}} M^{*} \cong P_{L^{*}} \otimes_{B} M^{*} \cong P_{L^{*}} \otimes_{B} P_{M^{*}}$. Since $k$ is a field, every tensor product of projective bimodules is a projective bimodule. This means that the complex $P_{L^{*}} \otimes_{B} P_{M^{*}}$ is a projective bimodule resolution of $L^{*} \otimes_{B}^{\mathbb{L}} M^{*}$.

Now we have shown that we can replace all the complexes in the diagrams by their projective resolutions and all the derived tensor functors by ordinary tensor functors, which are associative.

\section{Support VARIETIES}

In this section we recall the definition of support varieties of bounded complexes over a finite dimensional $k$-algebra $A$ using Hochschild cohomology, and we prove the main result as stated in the introduction. In the theory of support varieties, it is standard to work with classical varieties instead of schemes. Henceforth, we therefore assume our ground field $k$ to be algebraically closed.

We use a description of support varieties in terms of the derived category. This description comes from the as yet unpublished paper [BKSS15]. A short summary can be found in [Sol06, Section 10], from which we recall the definitions and results we need.

To define support varieties we need to recall the action of $\operatorname{HH}^{*}(A)$ on $\operatorname{Hom}_{\mathcal{D}^{b}(A)}\left(M^{*}, N^{*}\right)$ for complexes $M^{*}$ and $N^{*}$ in $\mathcal{D}^{b}(A)$. Define $\varphi_{M^{*}}: \operatorname{HH}^{*}(A) \rightarrow \operatorname{End}_{\mathcal{D}^{b}(A)}^{*}\left(M^{*}\right)$ as $\varphi_{M^{*}}=$ $-\otimes_{A}^{\mathbb{L}} M^{*}$. This is a homomorphism of graded rings and the left action of $\operatorname{End}_{\mathcal{D}^{b}(A)}^{*}\left(N^{*}\right)$ on 
$\operatorname{Hom}_{\mathcal{D}^{b}(A)}^{*}\left(M^{*}, N^{*}\right)$ induces a left action of $\operatorname{HH}^{*}(A)$ on this space. In order for an algebra $A$ to have a well-behaved support variety theory, the following two conditions need to be satisfied:

Definition 3.1. Let $A$ be a $k$-algebra. Let $H \subseteq H^{*}(A)$ be a subalgebra with $H^{0}=$ $\mathrm{HH}^{0}(A)$. Then $A$ is said to satisfy $(\boldsymbol{F g})$ with respect to $H$ if the following two conditions hold:

(Fg1) The algebra $H$ is a noetherian ring.

(Fg2) $\operatorname{Hom}_{\mathcal{D}^{b}(A)}^{*}\left(M^{*}, N^{*}\right)$ is a finitely generated $H$-module for all bounded complexes $M^{*}$ and $N^{*}$ of $A$-modules.

We say that $A$ satisfies $(\boldsymbol{F g})$ if there exists $H$ such that $A$ satisfies $(\mathrm{Fg}$ ) with respect to $H$.

There are several different ways to describe the $(\mathrm{Fg})$ condition. For an algebra $A$, the following statements are equivalent:

(1) $A$ satisfies (Fg).

(2) $A$ satisfies $(\mathrm{Fg})$ with respect to some commutative subalgebra $H$ of $\mathrm{HH}^{*}(A)$.

(3) $A$ satisfies $(\mathrm{Fg})$ with respect to $\mathrm{HH}^{*}(A)$.

(4) $A$ satisfies (Fg) with respect to $\mathrm{HH}^{\mathrm{ev}}(A)$.

The equivalence of these statements was shown in [Sol06, Propositions 5.5-5.7] under the assumption that $H$ is commutative. Note that the proofs therein do not rely on this assumption. Hence, the statements (1)-(4) are equivalent. Furthermore, under the presence of (Fg1) it suffices to check (Fg2) for the stalk complex $M^{*}=N^{*}=A / \operatorname{rad}(A)$, see [Sol06, Proposition 10.3].

Definition 3.2. Let $A$ be a $k$-algebra which satisfies $(\mathrm{Fg})$, and let $H \subseteq \mathrm{HH}^{*}(A)$ be a commutative subalgebra of the Hochschild cohomology ring of $A$ such that $A$ satisfies (Fg) with respect to $H$. For a pair $\left(M^{*}, N^{*}\right)$ of bounded complexes of $A$-modules, the support variety $\mathcal{V}_{A}^{H}\left(M^{*}, N^{*}\right)$ is the maximal ideal spectrum $\max \operatorname{Spec}\left(H / \operatorname{Ann}_{H}\left(\operatorname{Hom}_{\mathcal{D}^{b}(A)}^{*}\left(M^{*}, N^{*}\right)\right)\right)$. Denote $\mathcal{V}_{A}^{H}\left(M^{*}, M^{*}\right)$ by $\mathcal{V}_{A}^{H}\left(M^{*}\right)$.

If an algebra $A$ satisfies the $(\mathrm{Fg})$ condition with respect to several different commutative subalgebras $H \subseteq \mathrm{HH}^{*}(A)$ of the Hochschild cohomology ring, then the support variety theory for $A$ may depend on the choice of $H$, as the following example illustrates.

Example 3.3. Let $A=\mathbb{C}[x, y] /\left(x^{2}, y^{2}\right)$. According to [ES11, Proposition 9.1] this algebra satisfies $(\mathrm{Fg})$ (with respect to $H^{\prime}:=\mathrm{HH}^{2 *}(A)$ ). Its Hochschild cohomology ring can be obtained as follows: In Hol00, Holm computed the Hochschild cohomology ring of $\mathbb{C}[X] /\left(X^{2}\right)$. He obtained that $\mathrm{HH}^{*}\left(\mathbb{C}[X] / X^{2}\right) \cong \mathbb{C}[s, t, u] /\left(s^{2}, t^{2}, 2 s u, u t\right)$ with $\operatorname{deg} s=0$, $\operatorname{deg} t=1$ and $\operatorname{deg} u=2$. The Hochschild cohomology ring of $A$ is the tensor product of two copies of the Hochschild cohomology ring of $\mathbb{C}[X] /\left(X^{2}\right)$, see e.g. [BO08, Corollary 4.8], i.e. $\mathrm{HH}^{2 *}(A) \cong \mathbb{C}[s, u, \tilde{s}, \tilde{u}] /\left(s^{2}, \tilde{s}^{2}, 2 s u, 2 \tilde{s} \tilde{u}\right)$. Therefore, $\mathcal{V}_{A}^{H^{\prime}}(\mathbb{C}) \cong \operatorname{maxSpec} \operatorname{HH}^{2 *}(A) \cong \mathbb{A}^{2}$, the complex plane. On the other hand, there is a $\mathbb{Z} /(2)$-action on $\operatorname{HH}^{2 *}(A)$ sending $u$ to $-u$ and $\tilde{u}$ to $-\tilde{u}$. The ring of invariants is $H=\mathbb{C}\left[s, u^{2}, \tilde{s}, \tilde{u}^{2}, u \tilde{u}\right] \subset H^{\prime}$. The algebra $A$ 
also satisfies $(\mathrm{Fg})$ with respect to $H$ : It is Noetherian and if $I$ is a finite generating set of $\operatorname{End}_{\mathcal{D}^{b}(A)}^{*}(M, M)$ for $\operatorname{HH}^{2 *}(A)$, then $I \cup u I \cup \tilde{u} I$ is a finite generating set of $\operatorname{End}_{\mathcal{D}^{b}(A)}^{*}(M, M)$ for $H$. As $H=\mathrm{HH}^{2 *}(A)^{\mathbb{Z} /(2)}$ is the ring of invariants, $\mathcal{V}_{A}^{H}(\mathbb{C}) \cong \operatorname{maxSpec} H \cong \mathbb{A}^{2} /(\mathbb{Z} /(2))$ is a Kleinian singularity. In particular, it is not isomorphic to $\mathbb{A}^{2}$ as a variety.

The definition of support varieties relies on the map $\varphi_{M^{*}}: \operatorname{HH}^{*}(A) \rightarrow \operatorname{End}_{\mathcal{D}^{b}(A)}\left(M^{*}\right)$ for every bounded complex $M^{*}$. In [Sol06, Subsection 10.1], Solberg gives an explicit description of this map instead of using derived functors. He then remarks (in Subsection 10.2) that this explicit description coincides with the definition of $\varphi_{M^{*}}$ we stated. There is, however, no proof of this fact in SSol06, Subsection 10.2]. In the following remark, we recall Solberg's explicit description and show that both approaches define the same map. For our purposes, it is most convenient to consider $\varphi_{M^{*}}$ in terms of the derived tensor functor. For performing concrete computations, however, the explicit description would be more convenient.

Remark 3.4. For giving the explicit description from [Sol06] we need a projective resolution $P^{*}: \cdots \rightarrow P_{1} \rightarrow P_{0} \rightarrow 0$ of $A$ as an $A^{e}$-module, together with a quasi-isomorphism $\varepsilon: P^{*} \rightarrow A$. Consider a homogeneous element of degree $n$ in $\operatorname{HH}^{*}(A)$. Such an element can be represented by a roof

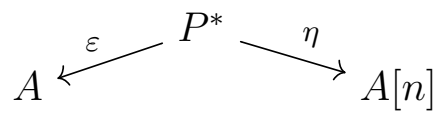

with the projective resolution $P^{*}$ on top and the quasi-isomorphism $\varepsilon$ as the left map. To get the result of the explicit description we apply $-\otimes_{A} M^{*}$ to this roof, and identify $A \otimes_{A} M^{*}$ with $M^{*}$. We obtain the the following roof:

$$
M^{*} \cong A \otimes_{A} M^{*} \stackrel{M^{*}}{\longleftarrow} P^{*} \otimes_{A} M^{*} \stackrel{\eta \otimes}{\longrightarrow} M^{*}
$$

This definition relies on the fact that the map $\varepsilon \otimes M^{*}$ is a quasi-isomorphism. We show this now. Since $k$ is a field, projective $A$-bimodules are projective as one sided $A$-modules. This implies that all the kernels of the sequence $\cdots \rightarrow P_{1} \rightarrow P_{0} \rightarrow A \rightarrow 0$ are projective as right $A$-modules. These are the same modules as the boundaries of the complex $P^{*}$. The assumptions of the Künneth formula [Rot09, Theorem 10.81] are therefore satisfied for the complex $P^{*} \otimes_{A} M^{*}$. They are also satisfied for the complex $A \otimes_{A} M^{*}$. From this we obtain the vertical isomorphisms in the following commutative diagram.

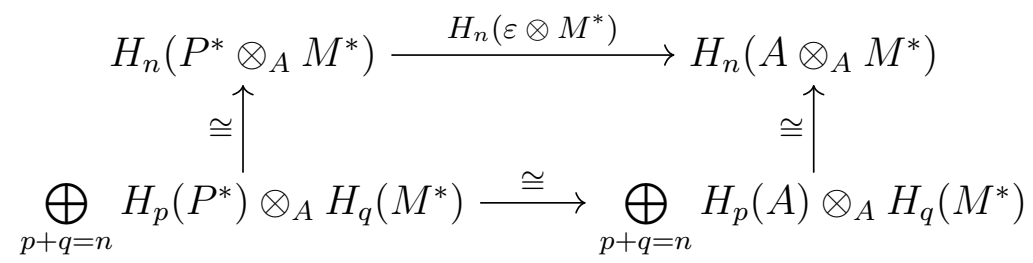

This shows that the map $\varepsilon \otimes M^{*}$ is a quasi-isomorphism. 
The following commutative diagram, where the bottom horizontal arrow is given by the explicit description, shows that the explicit description coincides with the description in terms of the derived tensor product $\varphi_{M^{*}}=-\otimes^{\mathbb{L}} M^{*}$ :

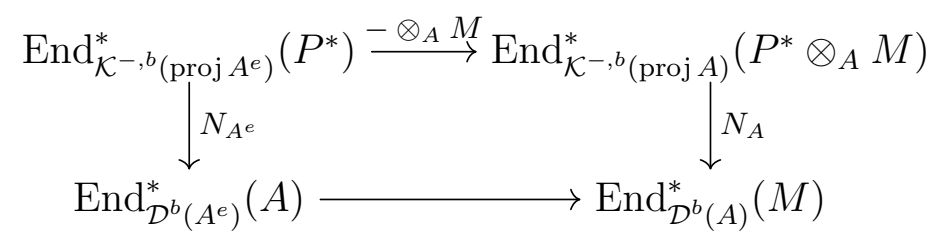

Now we are ready to prove our main result.

Theorem 3.5. Let $A$ and $B$ be derived equivalent $k$-algebras, and let ${ }_{B} X_{A}^{*}$ be a bimodule complex inducing a derived equivalence of standard type $X^{*} \otimes_{A}^{\mathbb{L}}-: \mathcal{D}^{b}(A) \rightarrow \mathcal{D}^{b}(B)$ with quasi-inverse given by the bimodule complex ${ }_{A} Y_{B}^{*}$. Let $f$ be the inverse of the isomorphism $\psi_{*} \circ\left(Y^{*} \otimes_{B}^{\mathbb{L}}\left(-\otimes_{B}^{\mathbb{L}} X^{*}\right)\right): \mathrm{HH}^{*}(B) \rightarrow \mathrm{HH}^{*}(A)$ given in Theorem 2.3. Then the following statements hold:

(1) For any bounded complex $S^{*}$ of A-modules, there is a commutative diagram

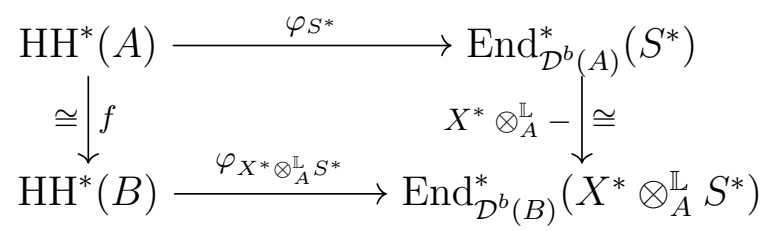

of graded rings, where the vertical maps are isomorphisms.

(2) Let $H$ be a subalgebra of $\mathrm{HH}^{*}(A)$. Let $H^{\prime}:=f(H)$. Then A satisfies (Fg) with respect to $H$ if and only if $B$ satisfies $(F g)$ with respect to $H^{\prime}$. In particular, $A$ satisfies (Fg) if and only if $B$ satisfies $(F g)$.

(3) Assume additionally that $H$ is commutative. Then, for bounded complexes $M^{*}$ and $N^{*}$ of A-modules, there is an isomorphism of varieties:

$$
\mathcal{V}_{A}^{H}\left(M^{*}, N^{*}\right) \cong \mathcal{V}_{B}^{H^{\prime}}\left(X^{*} \otimes_{A}^{\mathbb{L}} M^{*}, X^{*} \otimes_{A}^{\mathbb{L}} N^{*}\right)
$$

Proof. We first prove part (1) by constructing the following commutative diagram of graded rings:

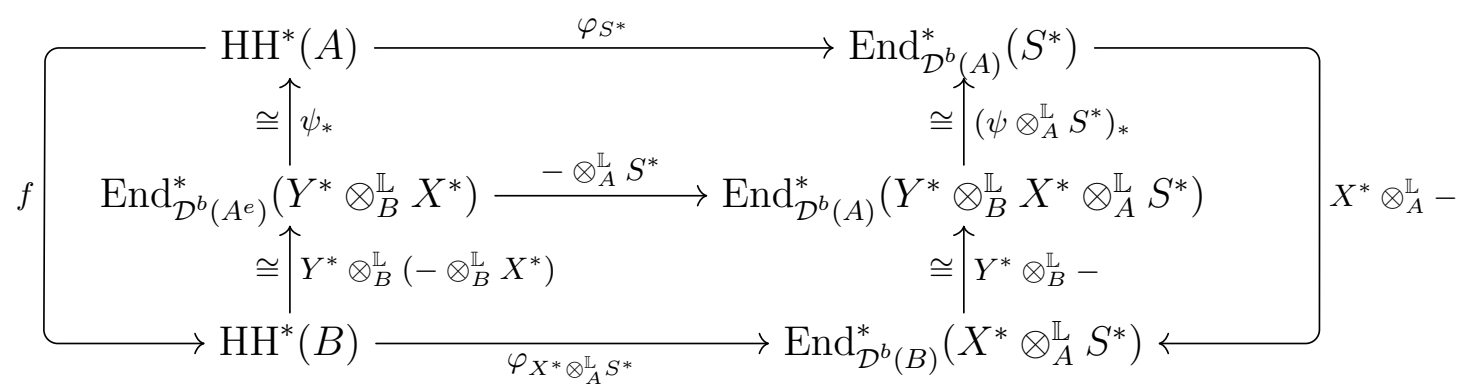

The associativity of the tensor product (Lemma 2.4) yields the commutativity of the lower part of the diagram. The upper square commutes since for $\eta \in \operatorname{End}_{\mathcal{D}^{b}(A)}^{*}\left(Y^{*} \otimes_{B}^{\mathbb{L}} X^{*}\right)$ there 
is the following chain of equalities:

$\left(\varphi_{S^{*}} \psi_{*}\right)(\eta)=\left(\psi \eta \psi^{-1}\right) \otimes_{A}^{\mathbb{L}} S^{*}=\left(\psi \otimes_{A}^{\mathbb{L}} S^{*}\right)\left(\eta \otimes_{A}^{\mathbb{L}} S^{*}\right)\left(\psi \otimes_{A}^{\mathbb{L}} S^{*}\right)^{-1}=\left(\psi \otimes_{A}^{\mathbb{L}} S^{*}\right)_{*}\left(\left(-\otimes_{A}^{\mathbb{L}} S^{*}\right)(\eta)\right)$.

To see that the triangle on the right part of the diagram commutes, start with a map $\alpha \in \operatorname{End}_{\mathcal{D}^{b}(A)}^{*}\left(S^{*}\right)$. Applying the maps $X^{*} \otimes_{A}^{\mathbb{L}}-$ and $Y^{*} \otimes_{B}^{\mathbb{L}}-$ gives the map $Y^{*} \otimes_{B}^{\mathbb{L}} X^{*} \otimes_{A}^{\mathbb{L}} \alpha$, and then by applying the map $\left(\psi \otimes_{A}^{\mathbb{L}} S^{*}\right)_{*}$, we get back to the original map $\alpha$.

We now prove parts (2) and (3). Consider the following diagram:

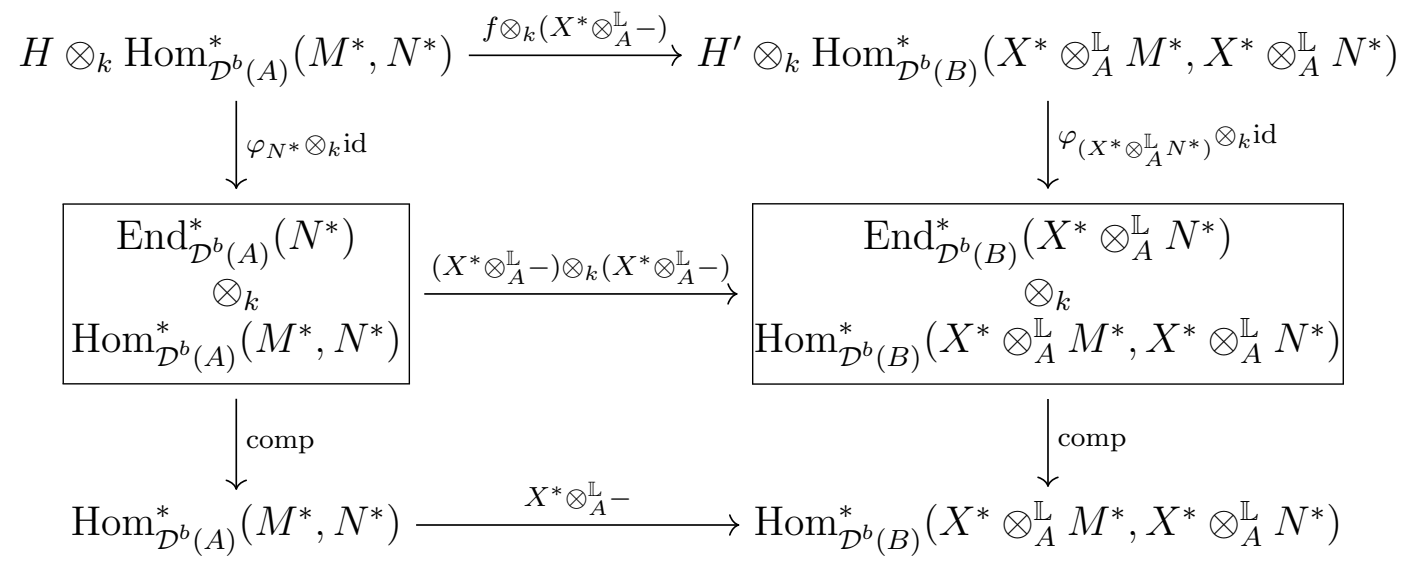

The upper square is commutative by part (1). The functoriality of $X^{*} \otimes_{A}^{\mathbb{L}}-$ implies that the lower square commutes. Since the horizontal arrows are isomorphisms it is straightforward to check that $\operatorname{Hom}_{\mathcal{D}^{b}(A)}^{*}\left(M^{*}, N^{*}\right)$ is finitely generated as an $H$-module if and only if $\operatorname{Hom}_{\mathcal{D}^{b}(B)}^{*}\left(X^{*} \otimes_{A}^{\mathbb{L}} M^{*}, X^{*} \otimes_{A}^{\mathbb{L}} N^{*}\right)$ is finitely generated as an $H^{\prime}$-module. It is also easy to see that the corresponding annihilators are isomorphic. Part (2) and (3) follow.

Remark 3.6. Let $F: \mathcal{D}^{b}(A) \rightarrow \mathcal{D}^{b}(B)$ be an arbitrary derived equivalence (not necessarily of standard type). By Theorem 2.2 , there is some equivalence of standard type

$$
X^{*} \otimes_{A}^{\mathbb{L}}-: \mathcal{D}^{b}(A) \rightarrow \mathcal{D}^{b}(B)
$$

which agrees with $F$ on objects. Hence, under the hypotheses of the foregoing theorem, there are isomorphisms

$$
\mathcal{V}_{A}^{H}\left(M^{*}, N^{*}\right) \cong \mathcal{V}_{B}^{H^{\prime}}\left(X^{*} \otimes_{A}^{\mathbb{L}} M^{*}, X^{*} \otimes_{A}^{\mathbb{L}} N^{*}\right) \cong \mathcal{V}_{B}^{H^{\prime}}\left(F\left(M^{*}\right), F\left(N^{*}\right)\right) .
$$

The subtlety here is that $H^{\prime}$ is not constructed directly using $F$ but going through determining a standard derived equivalence $X^{*} \otimes_{A}^{\mathbb{L}}-$ which agrees with $F$ on objects. This is not necessary if one sets $H=\mathrm{HH}^{\mathrm{ev}}(A)$ and $H^{\prime}=\mathrm{HH}^{\mathrm{ev}}(B)$. In this case, one has

$$
\mathcal{V}_{A}^{\mathrm{HH}^{\mathrm{ev}}(A)}\left(M^{*}, N^{*}\right) \cong \mathcal{V}_{B}^{\mathrm{H}{ }^{\mathrm{ev}}(B)}\left(F\left(M^{*}\right), F\left(N^{*}\right)\right) .
$$

Summing up, the (Fg) condition on Hochschild cohomology as well as the support variety theory are invariant under derived equivalences (of standard type). In the next section we will provide an instance of this theorem. 


\section{How to USE The TheOREM?}

In this section, we demonstrate how Theorem 3.5 can be used to produce examples of algebras which satisfy the (Fg) condition. We first summarize some earlier known results which are useful for determining whether a given algebra satisfies (Fg). Then we use some of these results together with Theorem 3.5 to discuss a new example of an algebra satisfying (Fg). Throughout this section, we let $k$ be an algebraically closed field.

As remarked in the introduction any algebra which satisfies (Fg) is a Gorenstein algebra (see $\left[\mathrm{EHS}^{+} 04\right.$, Proposition 1.2]). Recall that an algebra $A$ is Gorenstein, sometimes also called Iwanaga-Gorenstein, if $\operatorname{injdim}_{A} A<\infty$ and injdim $A_{A}<\infty$. For Nakayama algebras, the following result by Nagase reduces the problem of determining $(\mathrm{Fg})$ to the problem of determining Gorensteinness.

Theorem 4.1. Nag11, Corollary 10] Let $A$ be a Nakayama k-algebra. Then A satisfies the $(\mathrm{Fg})$ condition if and only if $A$ is a Gorenstein algebra.

Furthermore, there exists a concrete algorithm by Ringel Rin13 for determining whether a Nakayama algebra is Gorenstein. For Nakayama algebras with at most three simples, there is also a method by Chen and Ye CY14 for determining Gorensteinness. Thus, for Nakayama algebras, the problem of determining $(\mathrm{Fg})$ is completely solved, in the sense that there exists an algorithm for determining whether any given Nakayama algebra satisfies $(\mathrm{Fg})$.

The following result shows that in certain situations, the problem of determining whether $(\mathrm{Fg})$ is satisfied for an algebra $A$ can be reduced to the same problem for the smaller algebra $e A e$, where $e$ is some idempotent in $A$.

Theorem 4.2. [PSS14, Theorem 8.1 (i),(iv)] Let $A$ be a k-algebra, let $e$ be an idempotent in $A$, and let $B=A /\langle e\rangle$. Assume that $\operatorname{projdim}_{A}(B / \operatorname{rad}(B))<\infty$ and $\operatorname{projdim}_{(e A e)^{\text {op }}} A e<$ $\infty$. Then A satisfies the ( $F g$ ) condition if and only if eAe satisfies the ( $F g)$ condition.

For simplicity, we only consider derived equivalences induced by tilting modules in our example. By a result of Happel [Hap87, if $T$ is a tilting module, then $T \otimes_{A}^{\mathbb{L}}-: \mathcal{D}^{b}(A) \rightarrow$ $\mathcal{D}^{b}\left(\operatorname{End}_{A}(T)^{\mathrm{op}}\right)$ is a derived equivalence. We recall the definition of a tilting module:

Definition 4.3. Let $A$ be a $k$-algebra, and let $T$ be an $A$-module. Consider the following conditions on $T$ :

(i) $\operatorname{projdim}_{A} T<\infty$.

(ii) $\operatorname{Ext}_{A}^{i}(T, T)=0$ for every $i>0$.

(iii) There exists an exact sequence $0 \rightarrow A \rightarrow T_{0} \rightarrow \cdots \rightarrow T_{m} \rightarrow 0$ of $A$-modules where every $T_{i}$ is in add $T$.

If all these conditions are true, then the module $T$ is called a tilting module. If conditions (i) and (ii) are true, and the number of nonisomorphic direct summands of $T$ is $n-1$, where $n$ is the number of simple $A$-modules (up to isomorphism), then $T$ is called an almost complete tilting module. If $T$ is an almost complete tilting module and $N \in \bmod A$ is a module such that $T \oplus N$ is a tilting module and add $T \cap$ add $N=0$, then $N$ is called a complement to $T$. 
In our example, we use the following result to find tilting modules. The analogue of this result for cotilting modules is stated in [BS98, Proposition 3.2]. We include a proof for the sake of completeness. For the notion of left approximations, we refer to [AR92.

Proposition 4.4. Let $A$ be a $k$-algebra, let $M$ be an almost complete tilting $A$-module, and let $X$ be an indecomposable complement to $M$. If $f: X \rightarrow E$ is a map which is both a left add $M$-approximation of $X$ and a monomorphism, then $M \oplus$ Coker $f$ is a tilting module.

Proof. Let $Y=$ Coker $f$. There is an exact sequence $\eta: 0 \rightarrow X \stackrel{f}{\rightarrow} E \rightarrow Y \rightarrow 0$. We check that the three requirements of Definition 4.3 are satisfied for the module $M \oplus Y$.

Since $M \oplus X$ is a tilting module, both $E \in$ add $M$ and $X$ have finite projective dimension. It follows that $M \oplus Y$ has finite projective dimension. For the second requirement, we apply the functor $\operatorname{Hom}_{A}(-, M)$ to the sequence $\eta$ and get a long exact sequence

$$
0 \rightarrow \operatorname{Hom}_{A}(Y, M) \rightarrow \operatorname{Hom}_{A}(E, M) \stackrel{f^{*}}{\rightarrow} \operatorname{Hom}_{A}(X, M) \rightarrow \operatorname{Ext}_{A}^{1}(Y, M) \rightarrow \cdots
$$

Since $M \oplus X$ is a tilting module, $E$ is in add $M$ and $f^{*}$ is an epimorphism, it follows that $\operatorname{Ext}_{A}^{i}(Y, M)=0$ for $i>0$. Similarly, we show that $\operatorname{Ext}_{A}^{i}(M, Y)$ and $\operatorname{Ext}_{A}^{i}(Y, Y)$ are zero for $i>0$. We infer that $\operatorname{Ext}_{A}^{i}(M \oplus Y, M \oplus Y)=0$ for $i>0$.

We now check the third requirement, i.e. we construct an exact sequence

$$
0 \rightarrow A \rightarrow T_{0}^{\prime} \rightarrow \cdots \rightarrow T_{m^{\prime}}^{\prime} \rightarrow 0
$$

of $A$-modules, where each $T_{i}^{\prime}$ lies in $\operatorname{add}(M \oplus Y)$. Since $M \oplus X$ is a tilting module, there exists an exact sequence $0 \rightarrow A \stackrel{\iota n}{\rightarrow} T_{0} \rightarrow \cdots \rightarrow T_{m} \rightarrow 0$ of $A$-modules, where each $T_{i}$ is in $\operatorname{add}(M \oplus X)$. Taking out all summands isomorphic to the indecomposable module $X$, we can decompose the module $T_{i}$ as $T_{i} \cong M_{i} \oplus X^{t_{i}}$, where $M_{i}$ is in add $M$ and $t_{i} \geq 0$ is an integer. Define the monomorphism $f_{i}: T_{i} \rightarrow M_{i} \oplus E^{t_{i}}$, by using the identity on $M_{i}$ and the map $f$ on each summand in $X^{t_{i}}$. To construct the exact sequence $(*)$, we start with the exact commutative diagram

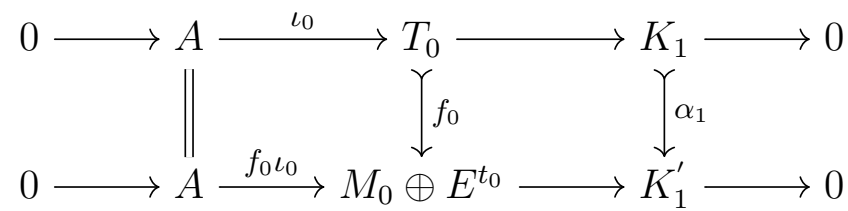

where $\alpha_{1}: K_{1} \rightarrow K_{1}^{\prime}$ is induced by the universal property of cokernels. Let $\iota_{1}: K_{1} \rightarrow T_{1}$ be the map induced by $T_{0} \rightarrow T_{1}$. Setting $T_{0}^{\prime}:=M_{0} \oplus E^{t_{0}}$ in the lower exact sequence of the above diagram, we get the first step of the sequence (*). Let $Y_{1}=$ Coker $\alpha_{1}$. Then $Y_{1} \cong$ Coker $f_{0} \in$ add $Y$. Taking the pushout of $\alpha_{1}$ along $f_{1} \iota_{1}$, we obtain the exact commutative diagram

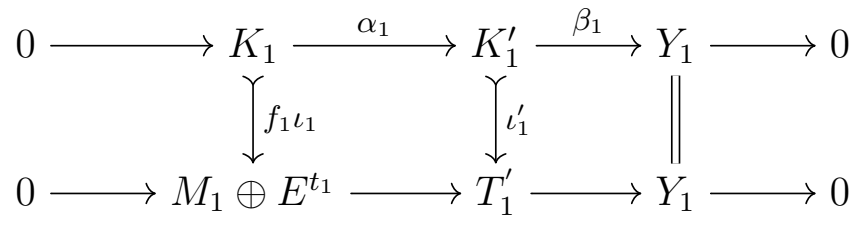


We know from above that $\operatorname{Ext}_{A}^{1}(Y, M)=0$. Since $Y_{1}$ is in add $Y$ and $M_{1} \oplus E^{t_{1}}$ is in add $M$, it follows that the bottom exact sequence of (4.4.1) splits, and thus $T_{1}^{\prime}$ is in $\operatorname{add}(M \oplus Y)$. Let $K_{2}^{\prime}=$ Coker $\iota_{1}^{\prime} \cong$ Coker $f_{1} \iota_{1}$. Hence, up to this point, we have constructed the following part of the exact sequence $(*)$ :

$$
0 \rightarrow A \rightarrow T_{0}^{\prime} \rightarrow T_{1}^{\prime} \rightarrow K_{2}^{\prime} \rightarrow 0
$$

with $T_{0}^{\prime}$ and $T_{1}^{\prime}$ in $\operatorname{add}(M \oplus Y)$. By applying the Snake Lemma to the exact commutative diagram

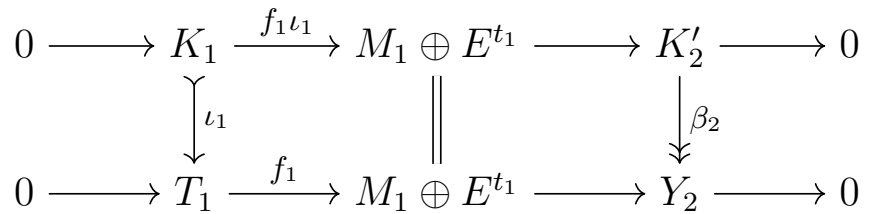

we get an exact sequence similar to the upper exact sequence of (4.4.1). The construction continues in the same way as above and the proof is finished.

Using the above results, we now construct two derived equivalent algebras, where one of the algebras is known to satisfy $(\mathrm{Fg})$. By Theorem 3.5, it follows that the other algebra also satisfies $(\mathrm{Fg})$.

Example 4.5. Let $A=k Q /\langle\rho\rangle$ be the $k$-algebra given by the following quiver and relations:

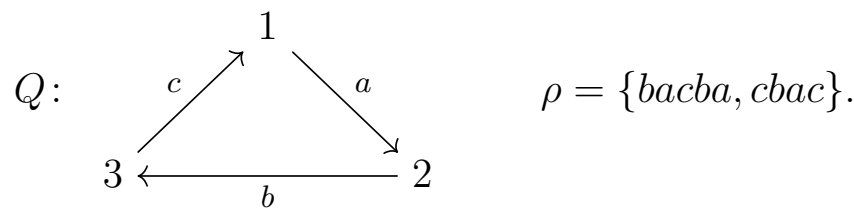

Then $A$ is a Nakayama algebra. The indecomposable projective modules are

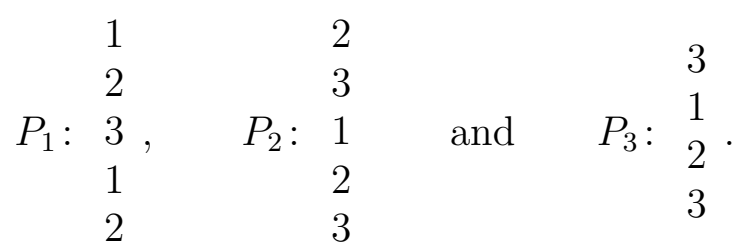

By [CY14, Proposition 3.14], this means that $A$ is a Gorenstein algebra (its normalized admissible sequence being $(4,5,5))$. Since $A$ is a Gorenstein Nakayama algebra, it satisfies (Fg) by Theorem 4.1 .

We now find an algebra which is derived equivalent to $A$. We use Proposition 4.4 to find a tilting module. Consider the almost complete tilting module $P_{1} \oplus P_{2}$ with complement $P_{3}$. The inclusion $P_{3} \hookrightarrow P_{2}$ is a left $\operatorname{add}\left(P_{1} \oplus P_{2}\right)$-approximation of $P_{3}$ and a monomorphism. By Proposition 4.4, the module $T=P_{1} \oplus P_{2} \oplus S_{2}$ is a tilting module.

We find the quiver and relations for the algebra $\operatorname{End}_{A}(T)$. The vertices of the quiver correspond to the three indecomposable summands $P_{1}, P_{2}$ and $S_{2}$ of $T$. The arrows of the 
quiver correspond to maps between these indecomposable modules. The following diagram depicts a $k$-basis for $\operatorname{End}_{A}\left(P_{1} \oplus P_{2} \oplus S_{2}\right)$ (except for the identity maps):

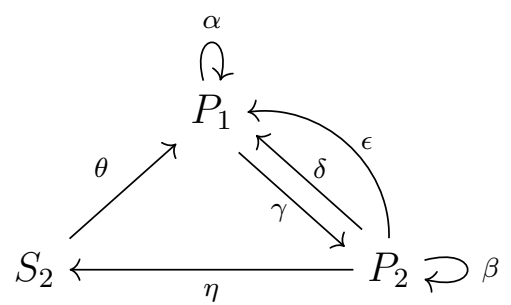

The maps are defined as follows:
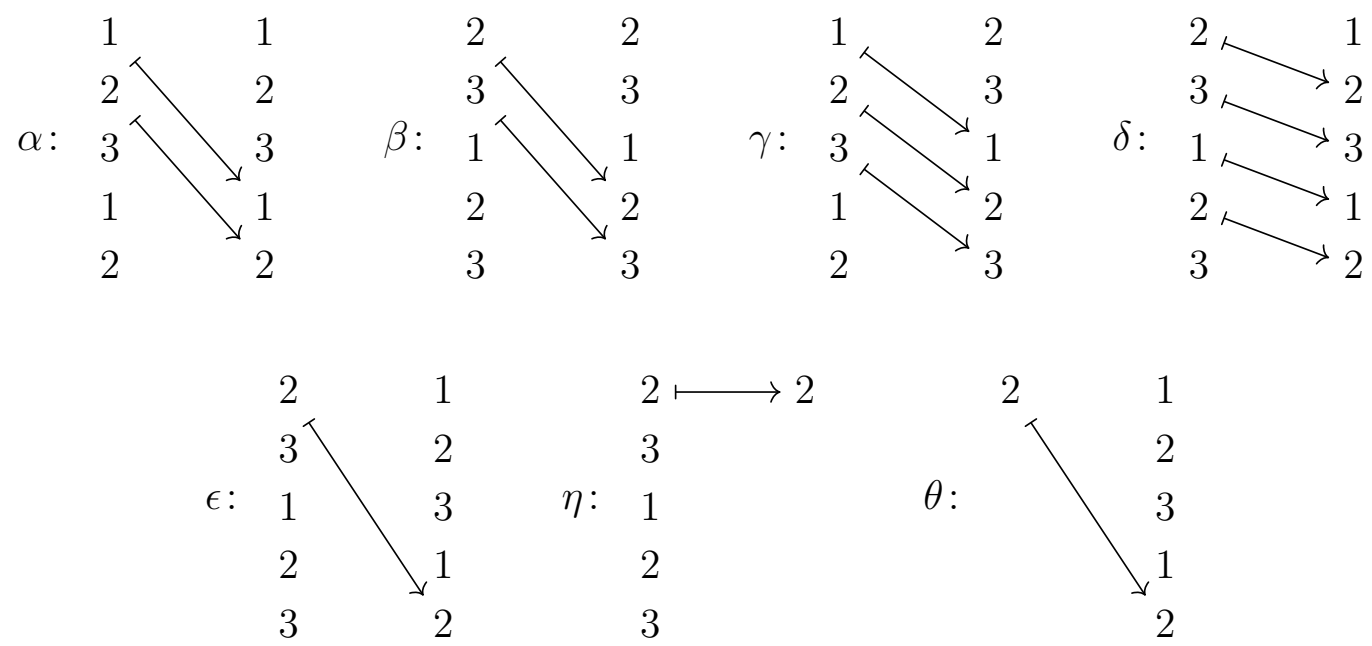

We have the following relations between these maps:

$$
\epsilon=\alpha \delta=\delta \beta=\theta \eta, \quad \alpha=\delta \gamma \quad \text { and } \quad \beta=\gamma \delta .
$$

We observe that the maps $\alpha, \beta$ and $\epsilon$ can be expressed in terms of other maps. By removing these maps from (4.5.1), we obtain the quiver of the algebra $\operatorname{End}_{A}(T)$. We find the relations of the algebra by using equations (4.5.2) and observing that the compositions $\gamma \delta \gamma, \eta \gamma$ and $\gamma \theta$ are zero. Let $Q^{\prime}$ and $\rho^{\prime}$ denote the quiver and relations

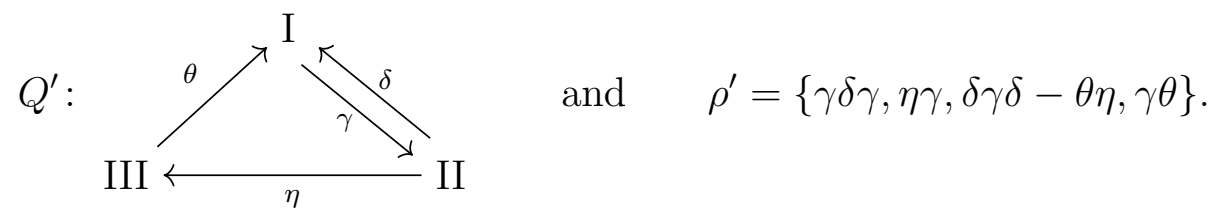

Then the endomorphism algebra $\operatorname{End}_{A}(T)$ must be a factor algebra of the algebra $k Q^{\prime} / \rho^{\prime}$. Furthermore, we observe that both these algebras have $k$-dimension 10 , so $\operatorname{End}_{A}(T) \cong$ $k Q^{\prime} / \rho^{\prime}$.

Let $B=\operatorname{End}_{A}(T)^{\text {op }}$. Then the algebras $A$ and $B$ are derived equivalent. Since $A$ satisfies the (Fg) condition, Theorem 3.5 tells us that $B$ also satisfies the (Fg) condition. 
We finally observe that we could not have used Theorem 4.2 to find out that the algebra $B$ satisfies (Fg). We can easily check that all the three simple $B$-modules have infinite projective dimension. This means that for any choice of nontrivial idempotent $e$ in $B$, the assumption of Theorem 4.2 is not satisfied. Therefore it is not possible to use this theorem to reduce the question of whether $(\mathrm{Fg})$ holds for $B$ to the same question about a smaller algebra $e B e$.

This example demonstrates that Theorem 3.5 can be used to show that (Fg) holds for an algebra which doesn't belong to one of the classes of algebras where $(\mathrm{Fg})$ is known to hold, e.g. group algebras, and for which other general theorems for deducing (Fg) do not apply.

\section{REFERENCES}

[AR92] Maurice Auslander and Idun Reiten. Homologically finite subcategories. In Representations of algebras and related topics (Kyoto, 1990), volume 168 of London Math. Soc. Lecture Note Ser., pages 1-42. Cambridge Univ. Press, Cambridge, 1992.

[BKSS15] Aslak Bakke Buan, Henning Krause, Nicole Snashall, and Øyvind Solberg. Axiomatic approach to support varieties. preprint in preparation, 2015.

[BO08] Petter Andreas Bergh and Steffen Oppermann. Cohomology of twisted tensor products. Journal of Algebra, 320(8):3327-3338, 2008.

[BS98] Aslak Bakke Buan and Øyvind Solberg. Relative cotilting theory and almost complete cotilting modules. In Algebras and modules, II (Geiranger, 1996), volume 24 of CMS Conference Proceedings, pages 77-92. American Mathematical Society, Providence, RI, 1998.

[Car83] Jon F. Carlson. The varieties and the cohomology ring of a module. Journal of Algebra, 85(1):104-143, 1983.

[CY14] Xiao-Wu Chen and Yu Ye. Retractions and Gorenstein homological properties. Algebras and Representation Theory, 17(3):713-733, 2014.

[EHS ${ }^{+}$04] Karin Erdmann, Miles Holloway, Nicole Snashall, Øyvind Solberg, and Rachel Taillefer. Support varieties for selfinjective algebras. $K$-Theory, 33(1):67-87, 2004.

[ES11] Karin Erdmann and Øyvind Solberg. Radical cube zero weakly symmetric algebras and support varieties. Journal of Pure and Applied Algebra, 215(2):185-200, 2011.

[Far07] Rolf Farnsteiner. Tameness and complexity of finite group schemes. Bulletin of the London Mathematical Society, 39(1):63-70, 2007.

[FP11] Eric Marc Friedlander and Julia Pevtsova. Constructions for infinitesimal group schemes. Transactions of the American Mathematical Society, 363(11):6007-6061, 2011.

[Hap87] Dieter Happel. On the derived category of a finite-dimensional algebra. Commentarii Mathematici Helvetici, 62(3):339-389, 1987.

[Hap91] Dieter Happel. On Gorenstein algebras. In Representation theory of finite groups and finitedimensional algebras (Bielefeld, 1991), volume 95 of Progress in Mathematics, pages 389-404. Birkhäuser, Basel, 1991.

[Hol00] Thorsten Holm. Hochschild cohomology rings of algebras $k[X] /(f)$. Beiträge zur Algebra und Geometrie. Contributions to Algebra and Geometry, 41(1):291-301, 2000.

[HTT08] Ryoshi Hotta, Kiyoshi Takeuchi, and Toshiyuki Tanisaki. D-modules, perverse sheaves, and representation theory, volume 236. Birkhäuser Boston, Inc., Boston, MA, 2008. Translated from the 1995 Japanese edition by Takeuchi.

[KZ98] Steffen König and Alexander Zimmermann. Derived equivalences for group rings, volume 1685 of Lecture Notes in Mathematics. Springer-Verlag, Berlin, 1998. With contributions by Bernhard Keller, Markus Linckelmann, Jeremy Rickard and Raphaël Rouquier. 
[Lin11] Markus Linckelmann. Finite generation of Hochschild cohomology of Hecke algebras of finite classical type in characteristic zero. Bulletin of the London Mathematical Society, 43(5):871-885, 2011.

[Nag11] Hiroshi Nagase. Hochschild cohomology and Gorenstein Nakayama algebras. In Proceedings of the 43rd Symposium on Ring Theory and Representation Theory, pages 37-41. Symp. Ring Theory Represent. Theory Organ. Comm., Soja, 2011.

[PSS14] Chrysostomos Psaroudakis, Øystein Skartsæterhagen, and Øyvind Solberg. Gorenstein categories, singular equivalences and finite generation of cohomology rings in recollements. Transactions of the American Mathematical Society Series B, 1:45-95, 2014.

[Ric89] Jeremy Rickard. Morita theory for derived categories. Journal of the London Mathematical Society. Second Series, 39(3):436-456, 1989.

[Ric91] Jeremy Rickard. Derived equivalences as derived functors. Journal of the London Mathematical Society. Second Series, 43(1):37-48, 1991.

[Rin13] Claus Michael Ringel. The Gorenstein projective modules for the Nakayama algebras. I. Journal of Algebra, 385:241-261, 2013.

[Rot09] Joseph Jonah Rotman. An introduction to homological algebra. Universitext. Springer, New York, second edition, 2009.

[Ska16] Øystein Skartsæterhagen. Singular equivalence and the (Fg) condition. Journal of Algebra, 452:66-93, 2016.

[Sol06] Øyvind Solberg. Support varieties for modules and complexes. In Trends in representation theory of algebras and related topics, volume 406 of Contemporary Mathematics, pages 239-270. American Mathematical Society, Providence, RI, 2006.

[SS04] Nicole Snashall and Øyvind Solberg. Support varieties and Hochschild cohomology rings. Proceedings of the London Mathematical Society. Third Series, 88(3):705-732, 2004.

[Wan14] Zhengfang Wang. Singular equivalence of Morita type with level. arXiv:1410.3140, 2014.

[Wei94] Charles A. Weibel. An introduction to homological algebra, volume 38 of Cambridge Studies in Advanced Mathematics. Cambridge University Press, Cambridge, 1994.

[Zim14] Alexander Zimmermann. Representation Theory: A Homological Algebra Point of View, volume 19 of Algebra and Applications. Springer Verlag, 2014.

Julian Külshammer, Institute of Algebra and Number Theory, University of Stuttgart, Pfaffenwaldring 57, 70569 Stuttgart, Germany

E-mail address: kuelsha@mathematik.uni-stuttgart.de

Chrysostomos Psaroudakis, Department of Mathematical Sciences, Norwegian UniverSity of Science And Technology, 7491 Trondheim, Norway

E-mail address: chrysostomos.psaroudakis@math.ntnu.no

Øystein Skartseterhagen, Department of Mathematical Sciences, Norwegian UniverSity of Science And Technology, 7491 Trondheim, Norway

E-mail address: oystein.skartsaterhagen@math.ntnu.no 